\title{
SPHERES Tethered Formation Flight Testbed: Advancements in Enabling NASA's SPECS Mission
}

\author{
Soon-Jo Chung ${ }^{a}$, Danielle Adams ${ }^{a}$, Alvar Saenz-Otero ${ }^{a}$, Edmund Kong ${ }^{a}$; \\ David W. Miller ${ }^{a}$, David Leisawitz ${ }^{b}$, Enrico Lorenzini ${ }^{c}$, Steve Sell ${ }^{d}$ \\ ${ }^{a}$ MIT Space Systems Laboratory, Cambridge, MA 02139; \\ ${ }^{b}$ NASA Goddard Space Flight Center, Greenbelt, MD 20771; \\ ${ }^{c}$ Harvard-Smithonian Center for Astrophysics, Cambridge, MA 02138; \\ ${ }^{d}$ Payload Systems Inc., Cambridge, MA 02139
}

\begin{abstract}
This paper reports on efforts to control a tethered formation flight spacecraft array for NASA's SPECS mission using the SPHERES test-bed developed by the MIT Space Systems Laboratory. Specifically, advances in methodology and experimental results realized since the 2005 SPIE paper ${ }^{4}$ are emphasized. These include a new test-bed setup with a reaction wheel assembly, a novel relative attitude measurement system using force torque sensors, and modeling of non-ideal tethers to account for tether vibration modes. The nonlinear equations of motion of multi-vehicle tethered spacecraft with elastic flexible tethers are derived from Lagrange's equations. The controllability analysis indicates that both array resizing and spin-up are fully controllable by the reaction wheels and the tether motor, thereby saving thruster fuel consumption. Based upon this analysis, linear and nonlinear controllers have been successfully implemented on the tethered SPHERES testbed, and tested at the NASA MSFC's flat floor facility using two and three SPHERES configurations.
\end{abstract}

Keywords: Stellar interferometer, space tether, formation flight

\section{INTRODUCTION}

The SPHERES Tether project of the MIT Space Systems Lab (MIT-SSL) is working in support of NASA's SPECS (Submillimeter Probe of the Evolution of Cosmic Structure) ${ }^{1-3}$ mission. SPECS is proposed as a tethered formation flight interferometer that detects submillimeter-wavelength light from the early universe. Because no previous space science mission has used a tethered formation flight architecture, many technology development bridges need to be crossed. Specifically, we are using the SPHERES (Synchronize Position Hold Engage and Reorient Experimental Satellite $)^{4}$ formation flight testbed to perform experiments in controlling tethered spacecraft arrays. Meanwhile, we are performing other analyses on various aspects of the SPECS mission, including nonlinear tether dynamics, control and estimation techniques for coupled spacecraft arrays, and optimal trajectory determination.

\subsection{Overview of the SPECS Mission}

The current architecture anticipated for SPECS calls for a two-aperture Michelson interferometer. ${ }^{1}$ The two 4-meter apertures and a beam combiner would be arranged in a line connected by two tethers, with the combiner in the center (see Fig. 1). The tethers could be reeled in and out to achieve baselines between the two apertures of up to one kilometer. The telescope will observe light in the range between 40 and $640 \mu \mathrm{m}$, using cryogenically cooled detectors. By observing light at the far infrared/submillimeter (FIR/SMM) wavelengths, the SPECS mission aims to address questions about the synthesis of the first heavy elements, the formation of galaxies through the merger of smaller galaxies, and the dynamics of protostars and gaseous debris disks. ${ }^{1}$ The proposed orbit for the observatory is at the Earth-Sun Lagrangian L2 point, which offers numerous advantages. For instance, this allows the tethered flight to be relatively undisturbed by gravity gradients avoiding significant

Further author information: (Send correspondence to )

Prof. David W. Miller: E-mail:millerd@mit.edu, Telephone: +1 (617) 253-3288

Advances in Stellar Interferometry, edited by John D. Monnier,

Markus Schöller, William C. Danchi, Proc. of SPIE Vol. 6268,

62680B, (2006) - 0277-786X/06/\$15 - doi: 10.1117/12.670489

Proc. of SPIE Vol. 6268 62680B-1 


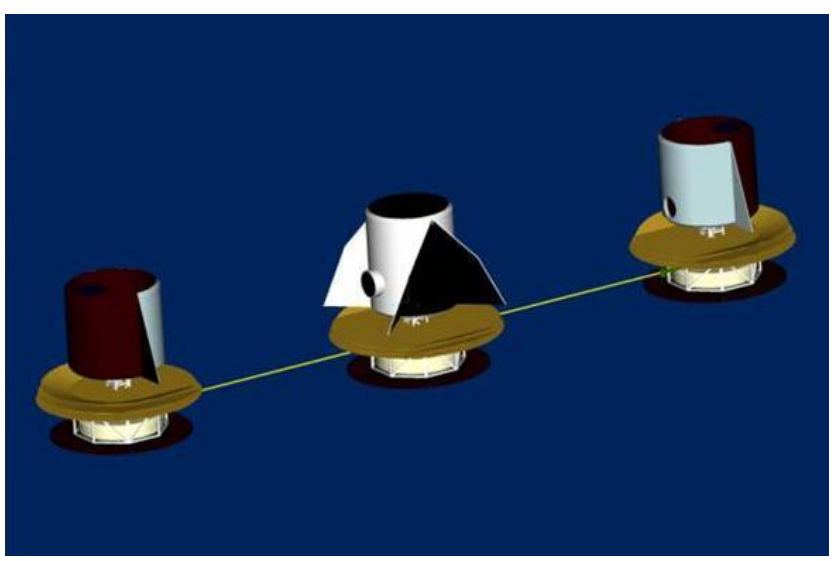

(a) SPECS

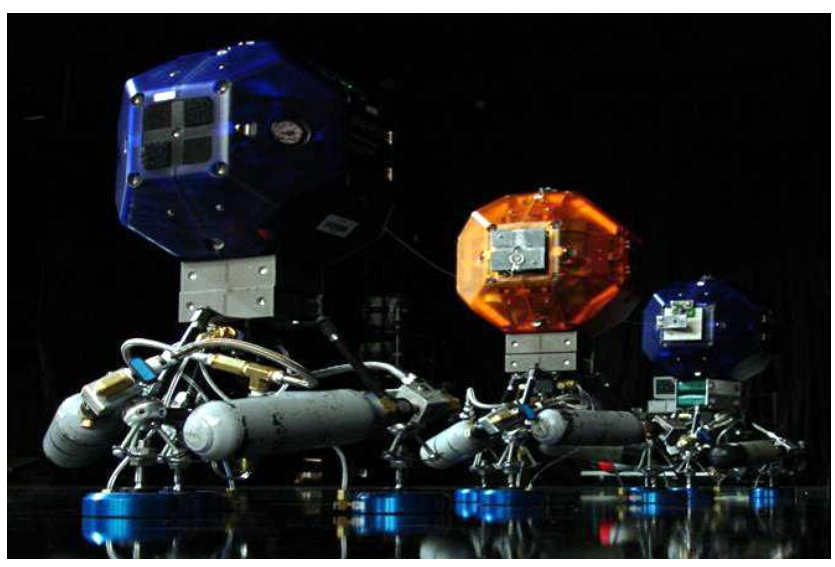

(b) SPHERES testbed on MSFC flat floor

Figure 1. Artistic rendering of the current configuration of $\operatorname{SPECS}^{2}$ (a) and SPHERES experimental setup (b)

wobbling of a spinning array. In addition, the L2 point provides a relatively unobstructed view of the sky and a lower thermal radiation background than a low-Earth orbit, which facilitates cryo-cooling. The basic observation scenario is to reel the tethers out or in gradually while rotating the array in a plane perpendicular to the target being observed. According to initial studies, each observation cycle performed in this way will take approximately 24 hours to achieve full UV plane coverage. ${ }^{2}$

\subsection{The Need for Tethered Formation Flight}

To achieve an unprecedented image resolution in space, a huge telescope is needed, since angular resolution is proportional to the wavelength divided by the telescope diameter. The Hubble Space Telescope (HST) is currently the largest spaceborne observatory having a diameter of $2.4 \mathrm{~m}$. Most current launch vehicles have fairing diameters between 3 and 5 meters, limiting the feasible size of a telescope aperture. SPECS aims at an angular resolution similar to that of HST, about 50 milliarcseconds. In the FIR/SMM wavelengths, this translates to a telescope diameter requirement on the order of 1 kilometer. ${ }^{2}$ Such a telescope could not be launched fully deployed. In addition, the fabrication cost of a large monolithic aperture is prohibitively expensive (recall Meinel's $l_{a w^{5}}$ ). To overcome these difficulties, stellar interferometry is currently being developed for space applications by NASA. Through interferometry, multiple apertures in large formation combine light coherently to achieve a fine angular resolution comparable to a filled aperture. SPECS will use tethered formation flight to achieve its large baseline requirements. The kinds of scenes that will be observed will contain information at many spatial frequencies, thus the ability of interferometers to observe at multiple baselines will be key. Tethered formation flight enables interferometric baseline changes with minimal fuel consumption; without tethers, a massive amount of fuel would be required to power the thrusters to change the baseline. The basic observational strategy can be easily achieved by reeling out the tethers and rotating the array in the plane of the tether. Compared to free-flying separated formation flight, this is a more efficient way to both know their relative positions and to control the spacecraft.

Tethered formation flight also presents its own challenges. It is expected that vibratory motion, consisting of compound pendulum modes of the satellite and tether violin modes, will be observed, affecting coherent interferometric beam combination. Highly maneuverable spacecraft are particularly problematic since beam control in the relay optics will need to be maintained to the requisite precision while thruster fire, tethers vibrate, and reaction wheels change momentum. However, the attitude control of tethered spacecraft will be responsible only for a fraction of the precision of the actual optical delay lines. As a rule of the thumb, controlling the locations of the apertures to within $10 \mathrm{~cm}$ is sufficient while the fine staged optical control maintains the Optical Path-length Difference (OPD) between individual apertures within a tenth of the operating wavelength. ${ }^{5}$ A longer wavelength of the FIR/SMM range makes SPECS even more technologically feasible. 


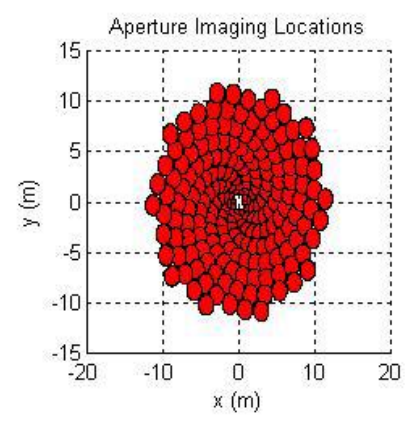

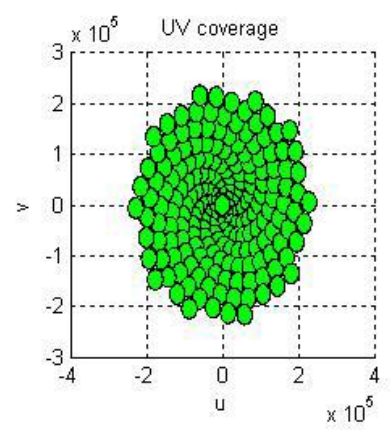

(a) constant angular rate mode
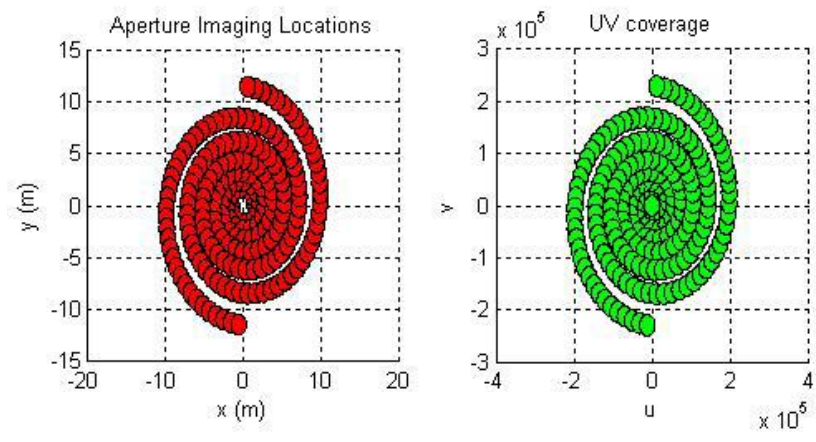

(b) constant linear velocity mode

Figure 2. Comparison of UV plots with the same initial velocities

\subsection{Open Issues in SPECS Development}

Several decisions are yet to be confirmed with respect to the SPECS architecture including the number of apertures and their configuration. ${ }^{1,2}$ As of the time of writing, the leading option is to have two light collectors at the ends of a line with a beam combiner in the middle as seen in Fig. 1. Another option is to have three light collectors at the vertices of a triangle and to have the beam combiner at some central location. Given a triangular configuration, there is the question of whether to use counterweights to avoid unwanted spin-up when the tether length changes. A second open issue is the method of spacecraft attitude actuation in order to control the spinning rate of the array as well as relative motions of each spacecraft. For now, the leading proposal is to use electric thrusters. The SPHERES team is analyzing the potential of actuation for in-plane (aperture pupil plane) rotation with only reaction wheels. This would significantly save the mass required for fuel to control the rotation rate of the array. A third open issue is the observation strategy. Given the basic goal of spiraling out and reaching some given level of UV plane coverage (i.e. more UV filling with smaller gaps in Modulation Transfer Function), there are several available strategies.

First, the array could rotate at constant angular momentum. In this case, an initial torque input starts the rotation then the angular rate decreases as the array reels out. The advantage of this strategy is that this mode can save thruster fuel consumption by exploiting the conservation of angular momentum. However, this would lead to extremely high values of uncontrolled rotation when the collectors are reeled in. The counterweight can be added to produce a more-slowly varying angular speed (e.g. Tetra-Star configuration ${ }^{3}$ ). A recent study ${ }^{6}$ investigated the feasibility of constant angular momentum spinning tethered formations for space-based interferometry applications in an Earth trailing, heliocentric orbit. Second, the array can be spun with constant thrust. This provides approximately constant angular velocity. ${ }^{2}$ Its high control authority yields the most desirable UV plane coverage, but the usage of propellant can be excessive.

Third, the array could rotate at constant linear velocity. This is useful for maintaining the same Signal to Noise Ratio (SNR) per baseline measurement since this mode ensures that the UV-point blur is constant throughout the imaging process. The constant linear velocity mode is proposed by the MIT study as a good compromise between economical thrust usage of the constant angular momentum mode and more effective UV plane coverage of the constant angular rate mode. Fig. 2 compares the UV plot of the constant linear velocity mode with that of the constant angular rate mode.

\subsection{Research Overview and Contributions}

In response to the open issues described above, the MIT SPHERES Tether project is focusing on the issue of actuation method and demonstration of controlled tethered formation flight. Specifically, the goal is to demonstrate experimentally that an array of tethered spacecraft can be controlled with only reaction wheels for actuating array spin rate $(\dot{\theta})$ and relative attitude between satellites $(\phi, \dot{\phi})$, defined in Fig. 5. A constant linear velocity mode will be compared with a constant angular rate mode to accentuate the economical benefit of the mode without losing acceptable UV plane coverage. 


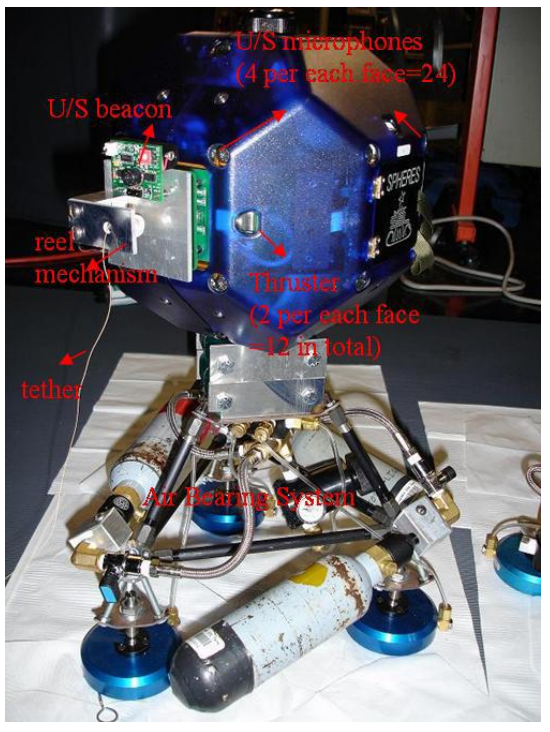

(a) SPHERES with previous aircarriage and tether reel

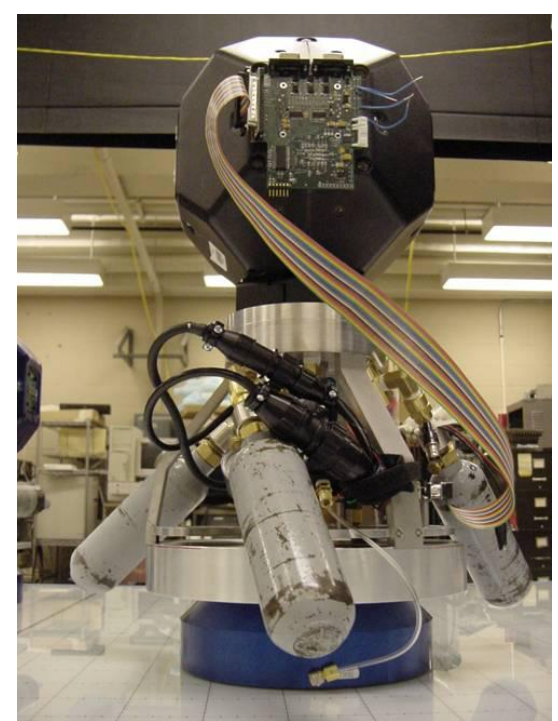

(b) SPHERES with new air-carriage and a reaction wheel

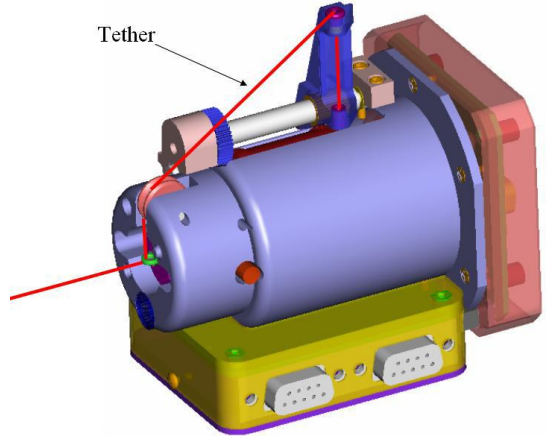

(c) CAD drawing of new tether mechanism under development

Figure 3. Evolution of the SPHERES tether experimental setup

Meanwhile, control strategies are being developed that do not require centralized and absolute knowledge of each spacecraft's position. Specifically, relative metrology is being used and each spacecraft is controlled independently using only information about relative distance and attitude from the adjacent spacecraft. We recently proved that a nonlinear control law stabilizing a singled-tethered spacecraft can also stabilize arbitrarily large circular arrays of spacecraft, as well as the three inline configuration using contraction theory. ${ }^{7}$ The importance of this approach lies in that we can employ a fully decentralized control from the reduced singletether system to control a more complex multiple-spacecraft array, reducing the complexity of both hardware and software. We refer the readers to the reference 7 for more details of the model reduction technique.

Furthermore, there are several advantages to the MIT strategy for actuation and control. First, using reaction wheels instead of thrusters for in-plane rotations means that power will be supplied via conversion of solar energy instead of by carrying expensive propellant. It is still envisioned to use thrusters for out-of-plane motions, but the life span of the mission would be greatly increased by using the reaction wheels for controlling the array spin-rate. Secondly, the optics will not risk contamination by exhaust from the thrusters. In addition, using decentralized control based on relative metrology means that the amount of communication required between spacecraft is reduced and the overall communication system can be simplified. This saves power and mass. The latest MIT strategies in metrology, control and dynamics modeling will be discussed below.

This paper is a follow up to the work published by Chung and Miller in 2005. ${ }^{4}$

\section{SPHERES TESTBED UPGRADES}

Recent improvements to the SPHERES Tether testbed have been made, including the design of a new flight quality tether reel, the use of a force-torque sensor to measure bearing angle and the building of an air carriage with a reaction wheel.

\subsection{SPHERES Overview}

The SPHERES testbed was developed as part of the ongoing research initiatives of the MIT-SSL that utilize the space environment provided by the International Space Station (ISS) to validate dynamics and control algorithms of distributed spacecraft control, estimation, and autonomy algorithms. The first batch of the SPHERES testbed 
was launched to the ISS on April 24th, 2006 for system identification and test of basic maneuvers in the 3dimensional environment. The operational environments also include the 2-dimensional flat floor facilities at MIT and the NASA Marshall Space Flight Center.

The individual self-contained satellites have the ability to maneuver in up to six Degrees of Freedom (DOF) (three rotations and three translations), to communicate with each other and with the laptop control station, and to identify their position and attitude with respect to each other and to the experiment global reference frame. Figure 3 exhibits a SPHERES satellite on the air-carriage for a planar 3-DOF test. The diameter of a single SPHERES is $0.25 \mathrm{~m}$, and the mass is $4.0 \mathrm{~kg}$. The satellites are propelled by a cold-gas thruster system which uses carbon dioxide as propellant. The CO2 propellant is stored in liquid form at 860 psig; a regulator reduces the pressure to 35 psig. Twelve thrusters are positioned to provide controllability in all six degrees of freedom, enabling both torque and translation control. Each thruster assembly, exerting a maximum of $0.12 \mathrm{~N}$ of force, consists of a solenoid-actuated micro-valve with machined nozzles. The SPHERES metrology system using the ultrasound system and gyroscope, provides metrology information to the satellites in real-time. Since no global metrology system like GPS is actually available in deep space missions, the tethered SPHERES system utilizes a relative metrology system using four ultrasound receivers (24 in total per each SPHERES satellite) on the line-of-sight face and the on-board beacon of the adjacent SPHERES (see Fig. 4). The relative metrology system is a pseudo-GPS ranging system that uses ultrasonic time-of-flight measurements from the target on-board beacon to the Ultrasound (U/S) microphones distributed on the surface of each satellite. These time-of-flight measurements are converted to ranges and are then used to derive relative attitude and rate $(\phi, \dot{\phi})$ with respect to the reference frame using a series of Extended Kalman Filters(EKF). ${ }^{4}$ An additional Kalman filter incorporating the gyroscope measurement estimates all the states (compound pendulum mode and array rotation rate) needed for each satellite. Each estimation algorithm is decentralized in the sense that it uses the single-tethered dynamics in Eq. (1). Texas Instruments C6701 Digital Signal Processor provides the computational power. A FLASH memory size of $224 \mathrm{~KB}$ allows software re-configuration of the full operating system, ensuring that multiple investigators are supported while the system is in the ISS. There is an expansion port which provides power and data interfaces to the auxiliary hardware. A tether deployment and retraction mechanism with tether forcetorque sensors has been added to this expansion port to support the tethered formation mission, which is detailed in the subsequent section.

\subsection{Flight Quality Tether Reel with Force-Torque Sensor}

The tether reel mechanism attaches to the SPHERES satellite via the expansion port, allowing for data and power transmission from the satellite (See. Fig. 3-(a)). The tether reel includes a motor and spool assembly to reel the tether in and out as well as a force/torque $(\mathrm{F} / \mathrm{T})$ sensor and ultrasound sensor to measure relative position and bearing of the neighbor satellite. A new flight tether reel is being developed for the ISS. Figure 3-(a) depicts the first tether reel attached to a SPHERES expansion port and (c) the CAD rendering of the new ISS-fight tether reel. The new version is designed to hold 10 meters of tether for flat floor testing. Some of the key design advantages of the new tether reel include an arm that moves along a shaft to ensure that the tether is evenly applied to the spool and prevent loss of tension. Also the reel assembly is very compact and includes both mechanical parts and the electronics boards to control them.

As described above, a $\mathrm{F} / \mathrm{T}$ sensor will be used to measure the bearing angle that the tether makes with respect to the satellite in the horizontal plane. Each SPHERES satellite needs to be able to measure this angle, portrayed as $\phi$ in Fig. 4. One of the goals of the controller is for this angle to be maintained at zero, thus minimizing the pendulum rotation mode. In the first experiments performed in $2004,{ }^{4}$ an ultrasound system was used to measure this angle as illustrated in Fig. 4-(a). With this system, a combination ultrasound beacon/receiver was positioned on each satellite directly facing a similar system on another satellite. In order to determine the distance between the satellites $(\ell)$ and the compound pendulum mode angle $(\phi)$, one satellite would send out a chirp to the second satellite, then the second satellite measures the time-of-flight measurements from four receivers on each face to calculate the range and attitude using the equations in Fig. 4-(a).

There are several reasons why a force/torque sensor will be used in the future to measure bearing angle in addition to the U/S system. The current U/S angle measurement system yields a one centimeter resolution. This results in merely a few degrees of resolution in bearing angle for $20 \mathrm{~cm}$ tether length. In addition, it does 


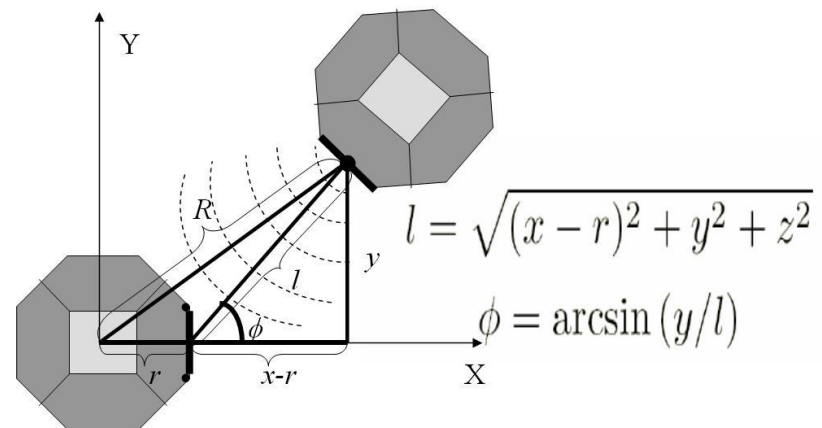

(a) Schematic of U/S system for measuring bearing angle $(\phi)$ and tether length $(\ell)$

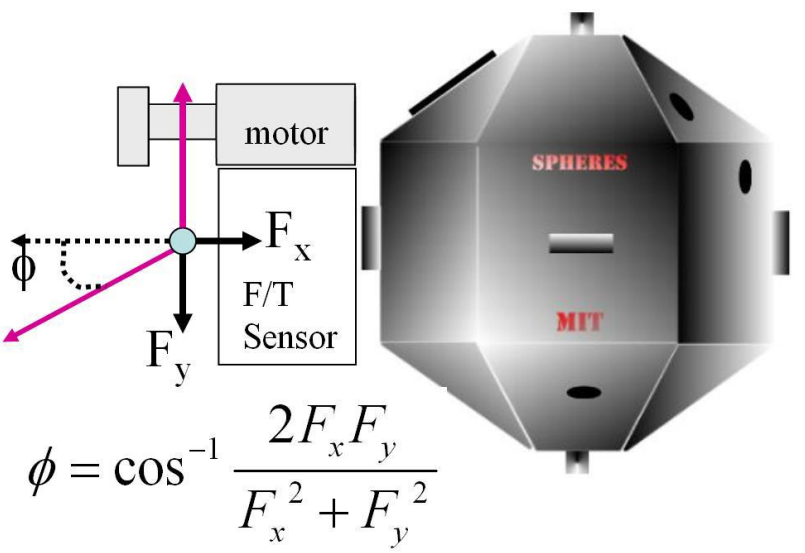

(b) Schematic of bearing angle measurement $(\phi)$ using $\mathrm{F} / \mathrm{T}$ output

Figure 4. Pendulum mode (bearing) angle measurement

not work well in close proximity due to the cone angle (+/- 30 degrees) of $\mathrm{U} / \mathrm{S}$ beacon. Experiments also reveal that measurements within $15 \mathrm{~cm}$ tend to be inaccurate. Moreover, it has a limitation in bandwidth. To avoid confusion, each SPHERES needs to identify the signal from a specific beacon number, separated by a time gap of $20 \mathrm{~ms}$. For three-SPHERES array, we can run this U/S metrology system at frequencies up to $10 \mathrm{~Hz}$. Also note that neither tether tension nor slackness can be measured by the current system.

The new bearing angle measurement system using force-torque $(\mathrm{F} / \mathrm{T})$ sensor outputs is under development to resolve those issues. The new $\mathrm{F} / \mathrm{T}$ sensor-based system simply compares two force outputs $\left(F_{x}\right.$ and $\left.F_{y}\right)$ to calculate the bearing angle $\phi$ using the equation given in Fig. 4-(b). The raw outputs from the $\mathrm{F} / \mathrm{T}$ sensors are sent to the FPGA circuitry board, which contains a 10 bit D/A for RWA control, two 6-channel A/D for force/torque measurements, and twelve $100-\mathrm{Hz}$ anti-aliasing filters. A series of EKFs then estimate all relevant states by mixing the $\mathrm{F} / \mathrm{T}$ output with high-bandwidth gyro measurement. ${ }^{4}$

\subsection{Reaction Wheel Air-Carriage}

Figure 3-(b) exhibits a SPHERES satellite mounted on a new air carriage with a reaction wheel embedded in its base. The satellites will be in a line as indicated Fig. 1 during experiments. The reaction wheels will be used to spin up the array of two or three satellites while the tether motor reels out the tether. There are two main operation modes for this spin up process. One is to spin up the array at constant rotation rate. In this case, the ratio of the initial and final angular momentum is $\frac{H_{o}}{H_{i}}=\frac{m l_{o}{ }^{2} \omega}{m l_{i}{ }^{2} \omega}=\frac{l_{o}{ }^{2}}{l_{i}{ }^{2}}$, where $l_{i}$ and $l_{o}$ denote the radius of the initial and final array baseline, respectively. The second operational mode is to spin up the array at constant linear velocity. The ratio of angular momentum is calculated as $\frac{H_{o}}{H_{i}}=\frac{m l_{o}\left(l_{o} \omega_{0}\right)}{m l_{i}\left(l_{i} \omega_{i}\right)}=\frac{l_{o}}{l_{i}}$ since $v=l_{o} \omega_{o}=l_{i} \omega_{i}=$ constant. Therefore, we can reduce the angular momentum requirement imposed on the reaction wheel assembly by a factor of $\frac{l_{o}}{l_{i}}$ via running at the constant linear speed mode.

During the process of sizing the reaction wheel, various scenarios were considered to spin up the array to various baseline lengths on either a flat floor or in the International Space Station. It was concluded that the most logical way to progress with the reaction wheel design was to start by building a system that only function on the flat floor and testing first with that. The current momentum wheel has an angular momentum capacity of $15 \mathrm{Nms}$ with the max torque rated at $0.318 \mathrm{Nm}$.

\section{DYNAMICS MODELING AND CONTROL STRATEGIES}

\subsection{Dynamics Modeling}

The equations of motion of a single-tethered system in Fig. 5 are presented in this section. This single tethered system is used as a basic model from which a more complex system is constructed. The tether is assumed to 


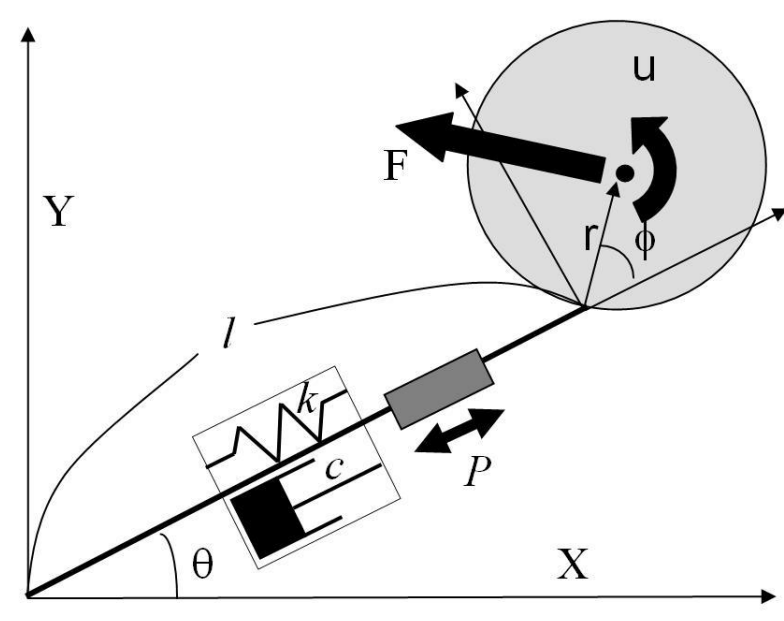

(a) Single tethered system

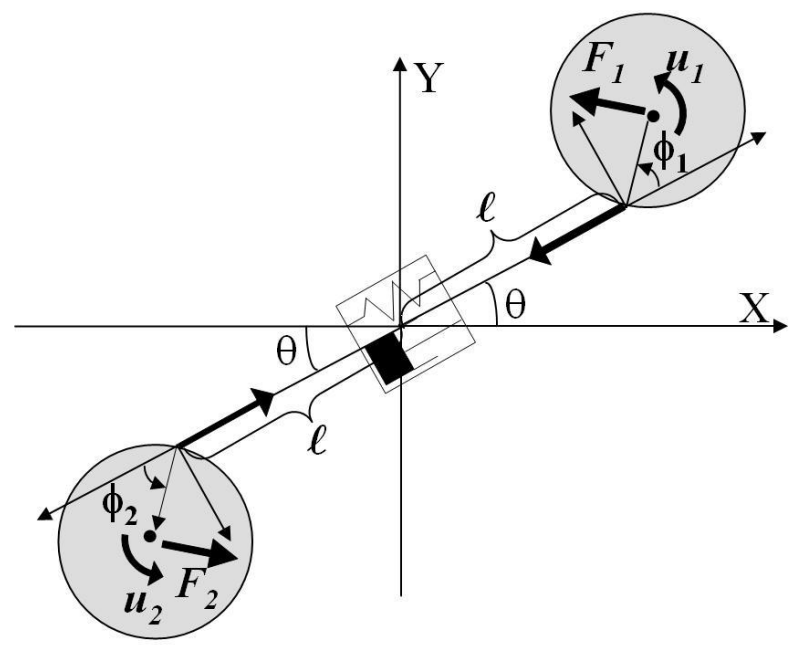

(b) Two spacecraft systems with two possible pendulum oscillations

Figure 5. Free-body diagram of single and two spacecraft systems

be massless, therefore, no transverse vibrations of tether (violin mode) are allowed unless noted otherwise. The zero mass assumption can be realized by strong thin material like Kevlar. At this point, the tether of SPECS consists of 4 tether lines, each with an oblate flattened cross section. ${ }^{2}$ It is estimated that the total mass of the 1-km-long ribbon tether will be less than $30 \mathrm{~kg}$. We later investigate a detrimental phenomenon such as the coupling between the violin mode of the massive tether and the spacecraft attitude dynamics such as the compound pendulum mode in Sect. 3.5. Additionally, the array is assumed to always rotate at a certain angular rate so the tether is taut and straight at all times. A nonzero angular rotation is a realistic assumption since tethered interferometers will attempt to fill a full $\mathrm{u}-\mathrm{v}$ coverage by rotation. The difference from the previous model in Ref.4 is that the tether is now extensible along its length, as depicted Fig. 5. Alternatively, this flexible tether can be regarded as a rigid tether connected in series with an elastic spring. The gravity model is neglected on the assumption that a tethered formation array such as SPECS will operate in a very weak gravity field - e.g. the second Lagrangian point L2 of the Earth-Sun system. Using Lagrange's equations, the following equations of motions on the array rotational plane (a plane normal to the aperture line-of-sight) are developed:

$$
\begin{aligned}
& \mathbf{M}_{\mathbf{1}}(\phi)\left(\begin{array}{l}
\ddot{\theta} \\
\ddot{\phi} \\
\ddot{\ell}
\end{array}\right)+\mathbf{C}_{\mathbf{1}}(\phi, \dot{\theta}, \dot{\phi})\left(\begin{array}{l}
\dot{\theta} \\
\dot{\phi} \\
\dot{\ell}
\end{array}\right)+\left(\begin{array}{lll}
0 & 0 & 0 \\
0 & 0 & 0 \\
0 & 0 & k
\end{array}\right)\left(\begin{array}{l}
\theta \\
\phi \\
\ell
\end{array}\right)=\left[\begin{array}{ccc}
r+\ell \cos \phi & 1 & 0 \\
r & 1 & 0 \\
0 & 0 & 1
\end{array}\right]\left(\begin{array}{l}
F \\
u \\
P
\end{array}\right) \\
& \text { where } \mathbf{M}_{\mathbf{1}}(\phi)=\left[\begin{array}{ccc}
I_{r}+m \ell^{2}+2 m r \ell \cos \phi & I_{r}+m r \ell \cos \phi & -m r \sin \phi \\
I_{r}+m r \ell \cos \phi & I_{r} & -m r \sin \phi \\
-m r \sin \phi & -m r \sin \phi & m
\end{array}\right] \text {, and } \\
& \mathbf{C}_{\mathbf{1}}(\phi, \dot{\theta}, \dot{\phi}, \dot{\ell})=\left[\begin{array}{ccc}
-m r \ell \sin \phi \dot{\phi}+m(r \cos \phi+\ell) \dot{\ell} & -m r \ell \sin \phi(\dot{\theta}+\dot{\phi}) & m(r \cos \phi+\ell) \dot{\theta} \\
+m r \ell \sin \phi \dot{\theta}+m r \cos \phi \dot{\ell} & 0 & m r \cos \phi \dot{\theta} \\
-m(r \cos \phi+\ell) \dot{\theta}-m r \cos \phi \dot{\phi} & -m r \cos \phi(\dot{\theta}+\dot{\phi}) & c
\end{array}\right] .
\end{aligned}
$$

In the equations above, $r, \ell$, and $I_{G}$ denote the satellite's radius, tether length, and moment of inertia. $I_{r}$ is the moment of inertia about the tether attachment point $\left(I_{r}=I_{G}+m r^{2}\right)$. The tether has both elasticity and damping modeled as a spring $k$ and a dashpot $c . F$ is the linear force due to thruster firing, and $u$ is the torque exerted on the Center of Mass (CM) of the satellite, e.g. torque by a Reaction Wheel Assembly (RWA). $P$ is the linear force actuated by the tether motor or the translational actuator. 
Note that this system reduces to the rigid-tether single-spacecraft system introduced in Ref. 4 and 7 if we assume that tether is not flexible $(c=0, k=0)$ and $\ell$ varies only at constant speed $(\ddot{\ell}=0)$, thereby eliminating the third equation of $\ddot{\ell}$ in Eq. (1).

This system has a rigid body mode of $\theta$ and undamped natural frequency of compound pendulum mode of $\omega_{\phi}=\sqrt{\frac{r\left(I_{r}+m \ell(2 r+\ell)\right)}{l I_{G}}} \omega[\mathrm{rad} / \mathrm{s}]$ when it is linearized about nominal velocities $\dot{\theta}=\omega, \dot{\ell}=v$ and $\dot{\phi}, \phi=0$. It can be shown that the system goes unstable when the tether motor reels in by checking the eigenvalues of the following Linear Time-Invariant (LTI) system,

$$
\frac{d}{d t}\left(\begin{array}{c}
\theta \\
\phi \\
\ell \\
\dot{\theta} \\
\dot{\phi} \\
\dot{\ell}
\end{array}\right)=\left[\begin{array}{cccccc}
0 & 0 & 0 & 1 & 0 & 0 \\
0 & 0 & 0 & 0 & 1 & 0 \\
0 & 0 & 0 & 0 & 0 & 1 \\
0 & \frac{r \omega^{2}\left(I_{r}+m r \ell\right)}{\ell I_{G}} & 0 & -2 \frac{v}{\ell} & 0 & -2 \frac{\omega}{\ell} \\
0 & -\frac{r \omega^{2}\left(I_{r}+m \ell(2 r+\ell)\right)}{\ell I_{G}} & 0 & 2 \frac{v}{\ell} & 0 & 2 \frac{\omega}{\ell} \\
0 & 0 & -\frac{k}{m} & 2(r+\ell) \omega & 2 r \omega & -\frac{c}{m}
\end{array}\right]\left(\begin{array}{c}
\theta \\
\phi \\
\ell \\
\dot{\theta} \\
\dot{\phi} \\
\dot{\ell}
\end{array}\right)+\left(\begin{array}{ccc}
0 & 0 & 0 \\
0 & 0 & 0 \\
0 & 0 & 0 \\
\frac{1}{m \ell} & -\frac{r}{I_{G} \ell} & 0 \\
-\frac{1}{m \ell} & \frac{r+\ell}{I_{G} \ell} & 0 \\
0 & 0 & \frac{1}{m}
\end{array}\right)\left(\begin{array}{c}
F \\
u \\
P
\end{array}\right)
$$

In other words, a positive reel-out speed $(v>0)$ resulted in damping of both $\dot{\theta}$ and pendulum motion of $\phi$ whereas we will see unstable motions of states for a negative reel-in speed $(v<0)$. Hence, it is indispensable to investigate this mode even for a large baseline length. It is also easy to verify that the system attitude states $(\dot{\theta}, \phi, \dot{\phi})$ with a nonzero $\omega$ are fully controllable even by $u$ only, using Eq. (2). ${ }^{4}$ In addition, $\ell$ is directly controllable by $P$. This controllability justifies our control approach to control the tethered array without using thrusters.

The nonzero rotational rate, $\omega$, added a potential term to the dynamics even though there is no gravitational force in the model. This nonzero artificial potential energy induced by the centrifugal force of array rotation, plays a crucial role in making the system controllable and stable, ${ }^{7}$ especially with $k=0$.

\subsection{Decentralized Control By Nonlinear Model Reduction}

The equations of motions of two-spacecraft array in Fig. 5-(b) can be shown to be the superposition of two singletethered system in Eq. (1). ${ }^{7}$ Derived by the Largrange's equation, Eq. (4) with $n=2$ represents the dynamics of a two-spacecraft array with constant $\dot{\ell}$ and inextensible tether. When linearized, this two-spacecraft system has a rigid body mode of $\dot{\theta}$. Additionally, it has the natural frequency of $\sqrt{\frac{r\left(I_{r}+m \ell(2 r+\ell)\right)}{l I_{G}}} \omega$ for synchronized $\phi$ and $\sqrt{\frac{m r \ell}{I_{r}}} \omega$ for anti-synchronized pendulum mode. Ref. 7 proved that both linear and nonlinear controllers designed from the single-tethered system as in Eq. (1) stabilized the two-spacecraft system, thereby enabling a fully decentralized control strategy. For example, consider the following decentralized linear control law for the two-spacecraft system given in Fig. 5.

$$
u_{i}=-K_{1} \phi_{I}-K_{2} \dot{\theta}_{i}-K_{3} \dot{\phi}_{i} \quad \mathrm{i}=1,2
$$

The stability condition of $K_{1}$ and $K_{3}$ for the anti-synchronized mode $\phi_{1}-\phi_{2}$ in Eq. (4) can be found as $K_{3}>0$ and $\omega_{o}^{2}+\frac{K_{1}}{I_{r}}>0$. Similarly, it can be shown that any controller satisfying $r K_{2}<(r+\ell) K_{3}, K_{1}>0, K_{2}>0$ can stabilize the rigid body mode of $\dot{\theta}$ and the synchronized mode of $\phi_{1}+\phi_{2}$. Note that $K_{3}>0$ and $\omega_{o}^{2}+\frac{K_{1}}{I_{r}}>0$ are automatically satisfied by this condition, thereby stabilizing the coupled two-body system in Fig. 5 -(b). Likewise, we can construct a nonlinear decentralized control law from the nonlinear dynamics equations of the single-system and its stability with global and exponential convergence can be proven using contraction theory. ${ }^{7}$

This reduction is applicable to any circularly symmetric array including a three triangular configuration. In this case, three imaginary pseudo-tethers connecting each satellite to the CM of the array are assumed to exist, replacing the three actual tether lines. When the tethers are taut and straight in a rotating array, a small $\phi$ (angle of the compound pendulum mode) is approximated as a perturbed angle that the satellites make with respect to the corresponding pseudo-tether. Here, $\ell$ is defined as the length of the pseudo-tether; the actual tether 
length is then $L=\sqrt{3}(\ell+r)-2 r$. The equations of motions of a three-spacecraft triangular array approximated by the pseudo-tethers are given in the following equation by setting $n=3$,

$$
\begin{gathered}
\mathbf{M}_{\mathbf{n}}(\mathbf{q})\left(\begin{array}{c}
\ddot{\theta} \\
\ddot{\phi_{1}} \\
\ddot{\phi}_{2} \\
\vdots \\
\ddot{\phi_{n}}
\end{array}\right)+\mathbf{C}_{\mathbf{n}}\left(\begin{array}{c}
\dot{\theta} \\
\dot{\phi}_{1} \\
\dot{\phi}_{2} \\
\vdots \\
\dot{\phi}_{n}
\end{array}\right)+\left(\begin{array}{c}
2 m\left(\sum_{k=1}^{n} r \cos \phi_{k}+\ell\right) \dot{\theta} \dot{\ell} \\
2 m r \cos \phi_{1} \dot{\theta} \dot{\ell} \\
2 m r \cos \phi_{2} \dot{\theta} \dot{\ell} \\
\vdots \\
\\
2 m r \cos \phi_{n} \dot{\theta} \dot{\ell}
\end{array}\right)=\left(\begin{array}{c}
\sum_{k=1}^{n} \tau_{\theta, k} \\
\tau_{\phi, 1} \\
\tau_{\phi, 2} \\
\vdots \\
\tau_{\phi, n}
\end{array}\right) \\
\text { where } \mathbf{M}_{\mathbf{n}}=\left[\begin{array}{ccccc}
\sum_{k=1}^{n} m_{11}\left(\phi_{k}\right) & m_{12}\left(\phi_{1}\right) & m_{12}\left(\phi_{2}\right) & \ldots & m_{12}\left(\phi_{n}\right) \\
m_{12}\left(\phi_{1}\right) & m_{22} & 0 & \ldots & 0 \\
m_{12}\left(\phi_{2}\right) & 0 & m_{22} & \ldots & 0 \\
\vdots & 0 & 0 & \ddots & 0 \\
m_{12}\left(\phi_{n}\right) & 0 & 0 & 0 & m_{22}
\end{array}\right], \\
\mathbf{C}_{\mathbf{n}}=\left[\begin{array}{ccccc}
\sum_{k=1}^{n} c_{11}\left(\phi_{k}, \dot{\phi}_{k}\right) & c_{12}\left(\phi_{1}, \dot{\theta}, \dot{\phi}_{1}\right) & c_{12}\left(\phi_{2}, \dot{\theta}, \dot{\phi}_{2}\right) & \ldots & c_{12}\left(\phi_{n}, \dot{\theta}, \dot{\phi_{n}}\right) \\
c_{21}\left(\phi_{1}, \dot{\theta}\right) & c_{22} & 0 & \ldots & 0 \\
c_{21}\left(\phi_{2}, \dot{\theta}\right) & 0 & c_{22} & \ldots & 0 \\
\vdots & 0 & 0 & \ddots & 0 \\
c_{21}\left(\phi_{n}, \dot{\theta}\right) & 0 & 0 & 0 & c_{22}
\end{array}\right]
\end{gathered}
$$

where $m_{i j}$ and $c_{i j}$ are defined as the (i,j) element of $M$ and $C$ matrices given from the single-tethered system in Eq. (1).

It is straightforward to show that a stabilizing controller for a single-tethered spacecraft will stabilize a threespacecraft triangular array. ${ }^{7}$ When linearized, the system has four eigenvalues, two of which are anti-synchrony mode. Eigenvalues for the triangular array $(n=3)$ are $0, \sqrt{\frac{r\left(I_{r}+m \ell(2 r+\ell)\right)}{l I_{G}}} \omega, \sqrt{\frac{m r \ell}{I_{r}}} \omega$, and $\sqrt{\frac{m r \ell}{I_{r}}} \omega$.

\subsection{Momentum Dumping Method for Saturated Wheels}

For satellites in orbit, thrusters are conventionally used to dump the angular momentum of the saturated momentum wheel. This section focuses on the issue associated with managing the saturated angular momentum once a tethered array spun by reaction wheels reaches its maximum size. A new technique that can be used to extend the array beyond this size is proposed. It is shown that a planar rotating array of tethered formation flight with an inextensible tether can control all relevant degrees of freedom using only one reaction wheel in each spacecraft. ${ }^{4}$ This result is easily extended to a model with an elastic tether as shown in Eq. (1) and (2). Due to the coriolis force exerted on the spacecraft, a radial motion of the tether can exert torque with respect to the compound pendulum mode $\phi$ in Fig. 5. Fast oscillatory motions of the tether then can be used as alternative means of controlling the pendulum mode. From Eq. (2), the dynamics of $\phi$ is coupled with $\dot{\ell}$ as $\ddot{\phi}+\frac{r \omega^{2}\left(I_{r}+m \ell(2 r+\ell)\right)}{\ell I_{G}} \dot{\phi}=2 \frac{v}{\ell} \dot{\theta}+2 \frac{\omega}{\ell} \dot{\ell}$. Since $\dot{\ell}$ is mainly driven by $P$, we can control $\phi$ by exerting $P$. This actuation method can be employed to dump the angular momentum stored on the reaction wheels. If the linear velocity or angular velocity of each spacecraft is held constant, the increase of the tether length inevitably leads to the saturation of the wheel speed. While constantly decelerating the wheel speed, a linear force, $P$ can be exerted in an oscillatory fashion to minimize the associated compound pendulum mode.

\subsection{Control Strategies for Three Inline Array}

Similarly, the dynamics of the three-spacecraft inline configuration in Figure 6 can be decoupled into two independent dynamics of the single-tethered system with the tether length $\ell$ and rotational dynamics of the center spacecraft. ${ }^{7}$ The equations of motions of the in-line array dynamics are developed using Lagrange's equation, 


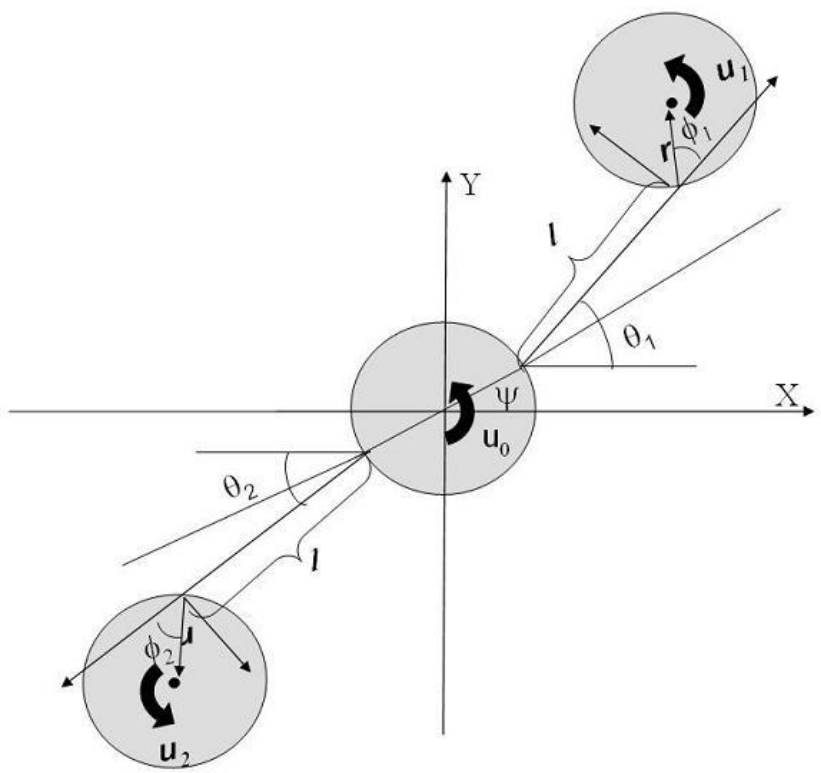

(a) Three-spacecraft inline-configuration
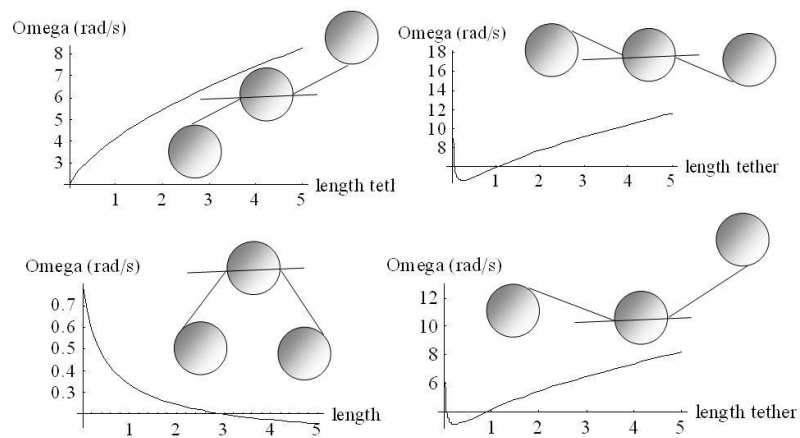

(b) Natural frequencies as a function of tether length with inextensible tether

Figure 6. Three inline configuration and its mode shapes

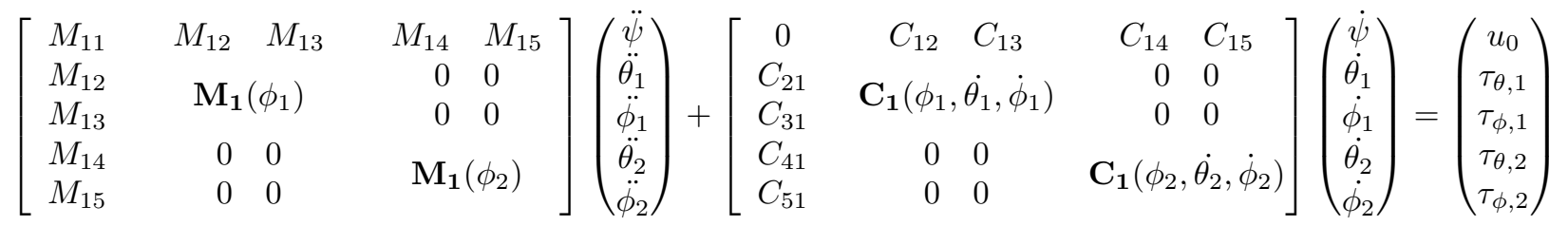

where $2 \times 2$ matrices, $\mathbf{M}_{\mathbf{1}}\left(\phi_{1}\right)$ and $\mathbf{C}_{\mathbf{1}}(\phi, \theta, \dot{\phi})$ are obtained from the single-tethered dynamics in figure 5 and Eq (1) by assuming $\dot{\ell}=0$. In addition,

$M_{11}=I_{r}=I_{G}+2 m r^{2}, M_{12}=m r \ell \cos \left(\theta_{1}-\psi\right)+m r^{2} \cos \left(\theta_{1}+\phi_{1}-\psi\right)$,

$M_{13}=m r^{2} \cos \left(\theta_{1}+\phi_{1}-\psi\right), M_{14}=m r \ell \cos \left(\theta_{2}-\psi\right)+m r^{2} \cos \left(\theta_{2}+\phi_{2}-\psi\right)$,

$M_{15}=m r^{2} \cos \left(\theta_{2}+\phi_{2}-\psi\right), C_{12}=-m r \ell \sin \left(\theta_{1}-\psi\right) \dot{\theta}_{1}-m r^{2} \sin \left(\theta_{1}+\phi_{1}-\psi\right)\left(\dot{\theta}_{1}+\dot{\phi}_{1}\right)$,

$C_{13}=-m r^{2} \sin \left(\theta_{1}+\phi_{1}-\psi\right)\left(\dot{\theta}_{1}+\dot{\phi_{1}}\right), C_{14}=-m r \ell \sin \left(\theta_{2}-\psi\right) \dot{\theta_{2}}-m r^{2} \sin \left(\theta_{2}+\phi_{2}-\psi\right)\left(\dot{\theta}_{2}+\dot{\phi}_{2}\right)$,

$C_{15}=-m r^{2} \sin \left(\theta_{2}+\phi_{2}-\psi\right)\left(\dot{\theta}_{2}+\dot{\phi}_{2}\right), C_{21}=m r \ell \sin \left(\theta_{1}-\psi\right) \dot{\psi}+m r^{2} \sin \left(\theta_{1}+\phi_{1}-\psi\right) \dot{\psi}$,

$C_{31}=m r^{2} \sin \left(\theta_{1}+\phi_{1}-\psi\right) \psi, C_{41}=m r \ell \sin \left(\theta_{2}-\psi\right) \dot{\psi}+m r^{2} \sin \left(\theta_{2}+\phi_{2}-\psi\right) \dot{\psi}, C_{51}=m r^{2} \sin \left(\theta_{2}+\phi_{2}-\psi\right) \dot{\psi}$.

When linearized, Eq. (5) yields four compound mode shapes, as depicted in Fig. 6-(b). The dynamics of the spacecraft at the tips of the array are shown to be a hierarchical combination since the dynamics get reduced to those of the single-tethered system if the dynamics of $\psi$ vanish. It is easy to implement an exponentially stabilizing controller for $\psi$, in particular with measurement of tether tensions. ${ }^{?}$ As long as $\psi, \dot{\psi} \rightarrow 0$ exponentially, the same controller stabilizing the single-tethered system in the previous sections will stabilize the combined system. The detailed proof of this section is presented in Ref. 7 .

\subsection{Tether Violin Mode}

Now we assume that the mass of the tether is not negligible. Especially in the case of the maximum tether deployment, up to $1 \mathrm{~km}$, a violin mode (transverse vibration of the string) is expected. Its interaction with other oscillatory mode such as the compound pendulum mode would be detrimental. In order to actively control this violin mode, we investigate an impedance matching ${ }^{8}$ control technique with a sliding tether boundary condition and a re-actuated transverse force shown in Fig. 7. Tether vibration is fundamentally governed by the wave 


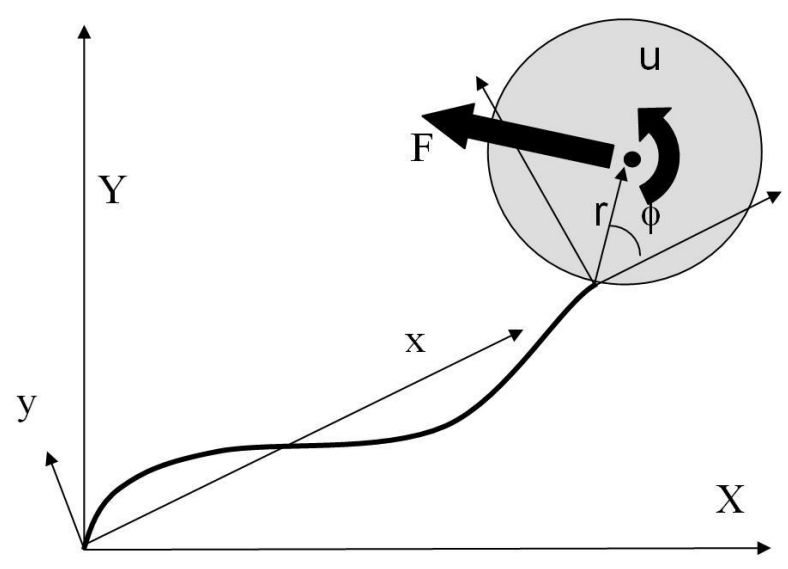

(a) Free-body diagram with massive tether

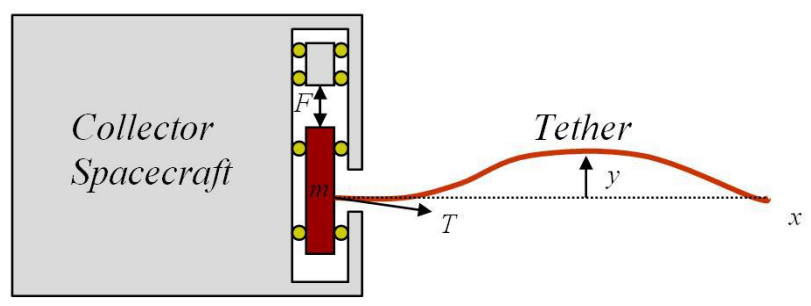

(b) Impedance Matched Tether termination

Figure 7. Modeling with massive tether

behavior of a contiguous string under tension (recall the wave equation: $\frac{\partial^{2} y}{\partial t^{2}}-\frac{T}{\rho A} \frac{\partial^{2} y}{\partial x^{2}}=0$ ). For each tether, motion can be decomposed into incoming $\left(w_{i}\right)$ and outgoing propagating waves $\left(w_{o}\right)$ resulting in $y=w_{i}+w_{o}$. The free-body diagram in Fig. (7)-(b) yields the force relation, $m \frac{\partial^{2} y}{\partial t^{2}}=\frac{\partial y}{\partial x}-F$. The boundary Ordinary Differential Equation (ODE), when transformed to wave coordinates, gives the following input-output condition.

$$
\left[\begin{array}{cc}
m \omega^{2} & T
\end{array}\right]\left[\begin{array}{cc}
1 & 1 \\
i k & -i k
\end{array}\right]\left(\begin{array}{c}
w_{i} \\
w_{o}
\end{array}\right)=F \Longrightarrow w_{o}=-\frac{m \omega^{2}+i k T}{m \omega^{2}-i k T} w_{i}+\frac{1}{m \omega^{2}-i k T} F
$$

Setting the outgoing wave to zero gives the force in terms of the incoming wave resulting in $F=\left(m \omega^{2}+i k T\right) w_{i}$. Transforming back to the physical coordinates gives the feedback law as the following,

$$
F=\frac{i \omega}{c_{o}} T y-m c_{o}(i \omega) \frac{\partial y}{\partial x}=\frac{T}{c_{o}} \frac{\partial y}{\partial t}-m c_{o}(i \omega) \frac{\partial^{2} y}{\partial x \partial t}
$$

Even though the current SPHERES testbed is not equipped with this kind of impedance matching actuator, simulation results will follow to see the effectiveness of such controller. Moreover, we are currently investigating the coupling motions ${ }^{9}$ of this tether violin mode with the spacecraft's attitude motion, in particular, the compound pendulum mode $(\phi, \dot{\phi})$ in Fig. 7 .

\section{EXPERIMENTAL RESULTS}

The Linear Quadratic Regulator (LQR) controller specifically addresses the issue of achieving a balance between good system response and the control effort required. Because the $A$ matrix in Eq. (2) is a function of $\omega$ and the tether length $\ell$, the optimal LQR gains are calculated over various angular rate and tether length. Since we are going to increase the tether length after achieving a certain angular rate, a gain-scheduled LQR gain is a continuous function of $\dot{\theta}$ over discrete tether length. Some of the curve-fitted functions resulted in a nonlinear quadratic function of $\dot{\theta}$. This gain-scheduled LQR controller has been successfully implemented in the SPHERES testbed. Figure 8 reveals the states background telemetry information directly recorded via the communication link from one of the satellites. The green line indicates the array angular rate $(\dot{\theta})$; blue is $\phi$; red is $\dot{\phi}$; light blue is gyro data. The second row of the figure represents the actuator output (the torque has a saturation limit at 0.012 $\mathrm{Nm}$ ). The LQR controller tried to spin up two tethered satellites into $0.3 \mathrm{rad} / \mathrm{s}$ from some arbitrary maneuvers. This control utilized only torque actuators to regulate the spin-up rate and to minimize the compound pendulum mode with the fixed tether length. The satellite encountered a sticky spot on the flat floor around 60-70 second interval, but shows a robust response back to the target angular rate. The constant torque actuation at the steady state indicates an existence of the surface friction which was not considered for linear modeling. 

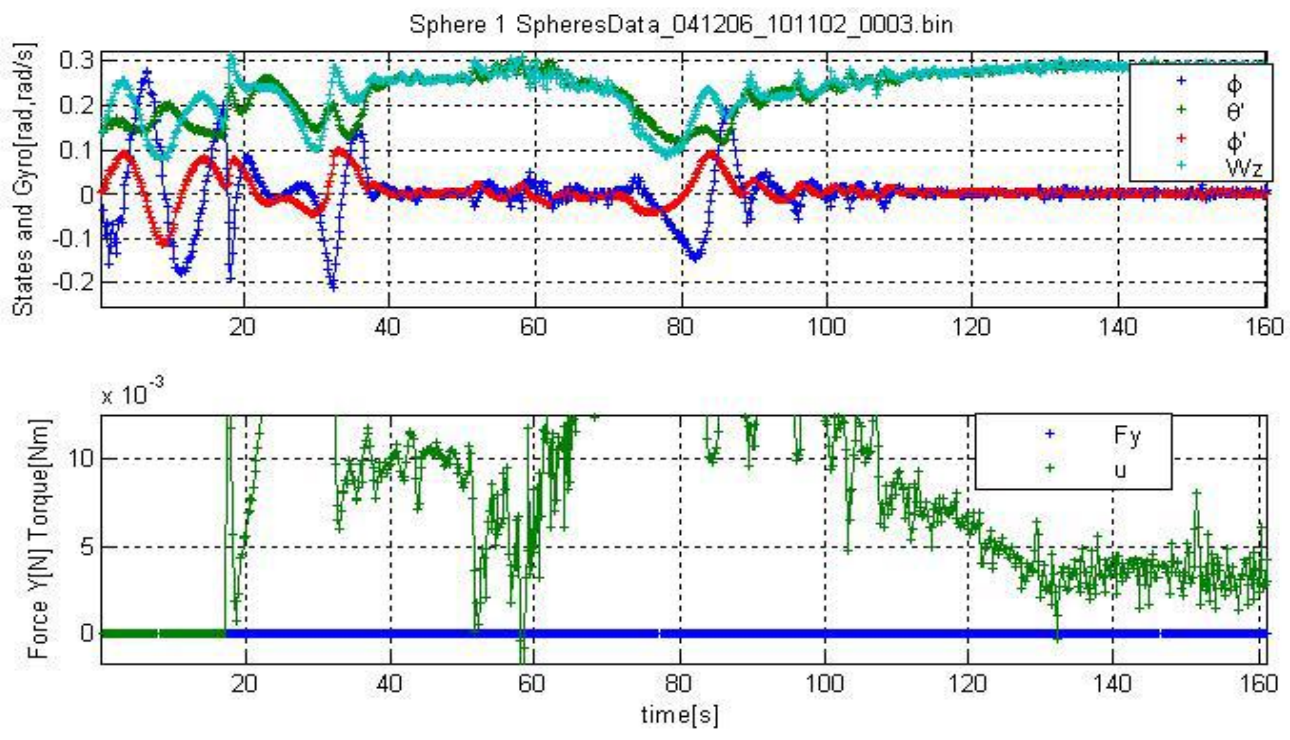

Figure 8. Gain-scheduled LQR controller in a singled-tethered system ${ }^{4}$

A nonlinear control approach based on Input-State Feedback Linearization is also employed when the system is fully actuated (i.e both thruster force $F$ and torque $u$ are available as control input). ${ }^{4}$ More experimental results are anticipated in the near future.

\section{CONCLUSION}

Tethered formation flight is being developed for future long-baseline space interferometers such as NASA's SPECS mission. This technique uniquely enables the acquisition of high-quality image data in a reasonable observing time for a given science target. This paper describes the ongoing efforts at MIT-SSL to experimentally validate some key control and estimation strategies for tethered formation flight, using multiple SPHERES microsatellites. In particular, two unique contributions of this research are identified as follows. First, we introduce a decentralized control technique that can be employed to reduce the complexity and dimensionality of the system. Decentralization is realized by nonlinear model reduction based upon oscillation synchronization. ${ }^{7}$ Second, we develop an underactuated nonlinear controller actuated only by reaction wheels, to control all relevant degrees of freedom, thereby minimizing thruster fuel consumption. Dynamics modeling of nonlinear tethered systems in various array configurations is also presented. Closed-loop control results using the new relative metrology system with force-torque sensors and air-carriage with a reaction wheel will follow.

\section{Acknowledgments}

The authors would like to gratefully acknowledge NASA for both financial and technical support for the MITSSL and PSI SPHERES Tether program. This work has been sponsored under NASA Phase II SBIR contract (Contract No.: NNG05CA09C).

\section{REFERENCES}

1. Harwit, M. "Kilometer-Baseline Far-Infrared/Submillimeter Interferometer Vision Mission: Final Report," Interferometer Vision Mission Team. May, 2005.

2. Lorenzini, E., Harwit, M., Bombardelli, C., Miller, D., Farley, R., Leisawitz, D., Rinehart, S., "The Role of Tethers in Far-Infrared/Submillimeter Astronomical Interferometry from Space," SPECS Final Report. May, 2005. 
3. Farley, R.E., and Quinn, D. A., "Tethered Formation Configurations: Meeting The Scientific Objectives Of Large Aperture And Interferometric Science," AIAA 2001-4770.

4. Soon-Jo Chung, Edmund M. Kong, and David W. Miller, "Dynamics and Control of Tethered Formation Flight Spacecraft Using the SPHERES Testbed," 2005 AIAA GNC, AIAA 2005-6089, San Francisco, August 2005.

5. Chung, S.-J., Miller, D. W., deWeck, O. L., "ARGOS Testbed: Study of Multidisciplinary Challenges of Future Spaceborne Interferometric Arrays," SPIE Optical Engineering, Vol. 43, No. 9, pages 2156-2167, September 2004.

6. Bombardelli, C., Lorenzini, E.C., and Quadrelli, M.B., "Formation Pointing Dynamics of Tether-Connected Architecture for Space Interferometry," The Journal of the Astronautical Sciences, Vol. 52, No. 4, October.December 2004, pp. 475.493.

7. Soon-Jo Chung, Jean-Jacques E. Slotine, and David W. Miller, "Nonlinear Model Reduction and Decentralized Control of Tethered Formation Flight," AIAA Journal of Guidance, Control and Dynamics. under review (also AIAA-2006-6589).

8. Miller, D. W., von Flotow, A. H., "A Traveling Wave Approach to Power Flow in Structural Networks," Journal of Sound and Vibration, 128(1), 145-162, 1989.

9. Bergamaschi, S., and Bonon, F., "Coupling of Tether Lateral Vibration and Subsatellite Attitude Motion," J. Guidance, Control, and Dynamics, Vol. 15, No, 5. 